\title{
THE RADIO SKY AT MICRO-JY LEVELS
}

\author{
E. B. FOMALONT \\ National Radio Astronomy Observatory \\ 520 Edgemont Road, Charlottesville, VA 22903, USA
}

\section{Introduction}

The properties of the $\mu \mathrm{Jy}$ radio sources are described in this paper. The results were obtained from deep VLA observations of four fields, shown in Table 1. Deep optical images have been obtained for the Bootes and Lilly1 fields.

TABLE 1. VLA DEEP OBSERVATIONS

\begin{tabular}{|c|c|c|c|c|c|c|c|c|}
\hline Field & \multicolumn{2}{|c|}{$\mathrm{J} 2000$} & $\begin{array}{l}\text { Freq } \\
\text { GHz }\end{array}$ & $\underset{\prime \prime}{\text { Res }}$ & $\begin{array}{c}\mathrm{S}_{l i m} \\
\mu \mathrm{J} y\end{array}$ & $\begin{array}{l}\text { Int } \\
\text { hrs }\end{array}$ & No & Ref \\
\hline Bootes & 141759 & $52 \quad 2712$ & $4.9,1.4$ & 4 & 16.0 & 126 & 62 & $(1),(2)$ \\
\hline Cepheus & 031725 & 802105 & 8.4 & 10 & 22.9 & 20 & 14 & (3) \\
\hline Lynx & 084504 & 443405 & 8.4 & 10 & 14.5 & 62 & 6 & (3) \\
\hline Lilly 1 & 131217 & 423806 & 8.4 & 6 & 7.0 & 159 & 39 & (4) \\
\hline
\end{tabular}

(1)=Fomalont et al. 1991, (2)=Hammer et al. 1995, (3)= Windhorst et al. 1993, (4)=Windhorst et al. 1995, and unpublished

\section{The Radio Source Properties}

The following radio properties are based on 121 sources in the complete samples. The Euclidean normalized counts of sources at $8.4 \mathrm{GHz}$, derived from the three $8.4 \mathrm{GHz}$ experiments, are shown in Figure 1. The best fit slope to the $8 \mathrm{GHz}$ counts at $\mu \mathrm{Jy}$ levels is $N=18 S^{-1.2}$, where $N$ is the number of sources (arcmin $)^{-2}$ with a flux density greater than $S \mu \mathrm{Jy}$. This corresponds to a differential count of $n=0.33 S^{-2.2}(\mathrm{Jy})^{-1}(\mathrm{sr})^{-1}$. The error of the count parameters is about $10 \%$. 


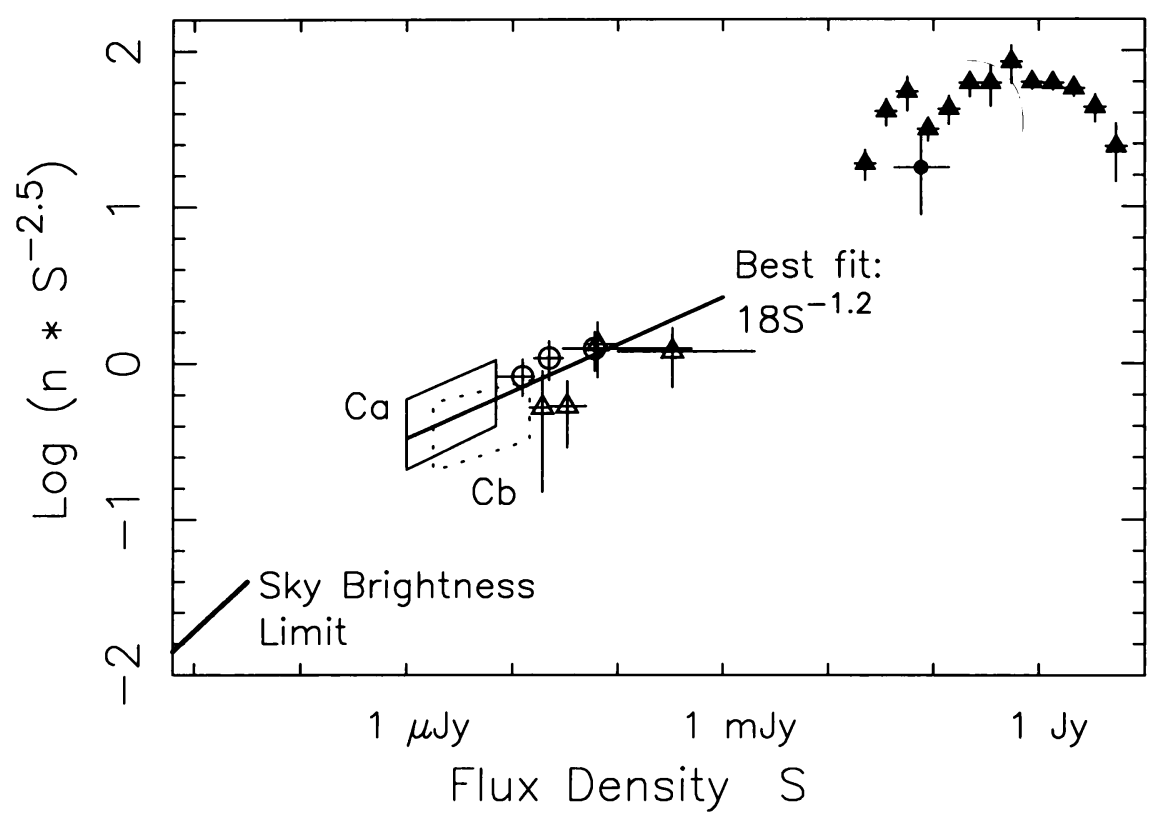

Figure 1. The Source Counts at $8.4 \mathrm{GHz}$ : The number, n, of sources $(\mathrm{Jy})^{-1}(\mathrm{sr})^{-1}$ is the ordinate. The plotted points show the count from the detected sources (Lynx and Cepheus fields $\triangle$, the Lilly 1 field $\bigcirc$ ). The trapezoidal boxes are the count derived from the fluctuations in the image caused by weak sources (Ca box for the Lynx and Cepheus fields, Cb box for the Lilly 1 field). See Windhorst et al. (1993). The best fit slope to the 8 GHz data $<1 \mathrm{mJy}$ is shown by the solid line. The upper limit source count at the nanoJy level which is consistent with less than $0.02 \mathrm{~K}$ sky brightness from discrete sources at 8 $\mathrm{GHz}$ is shown by the other solid line.

The slope, $\gamma=-2.2$, of the count is surprisingly steep and close to the Euclidean value of -2.5 . Since the typical redshift is $>0.5$ for the $\mu \mathrm{Jy}$ sources, they have evolved with cosmological epoch. However, detailed models cannot be made with the limited sample. An upper limit to the count of sources at the nanoJy levels can be obtained from measurements of the sky brightness. At frequencies between 3 to $50 \mathrm{GHz}$ there is no excess sky brightness contribution, above that of the CBR of $2.74 \mathrm{~K}$, at a level of $0.02 \mathrm{~K}$ (Windhorst et al., 1993). The line in the lower left of Figure 1 conforms to this limit.

The estimate of the average angular size of the $\mu \mathrm{Jy}$ radio sources have been determined using detailed simulations of the effects of the blending of faint sources (Richard 1996). Of the 38 sources in the Lilly1 field, nine were resolved at $6^{\prime \prime}$ resolution. However, the simulations show that about this number of sources should appear to be resolved, solely from confusion with 


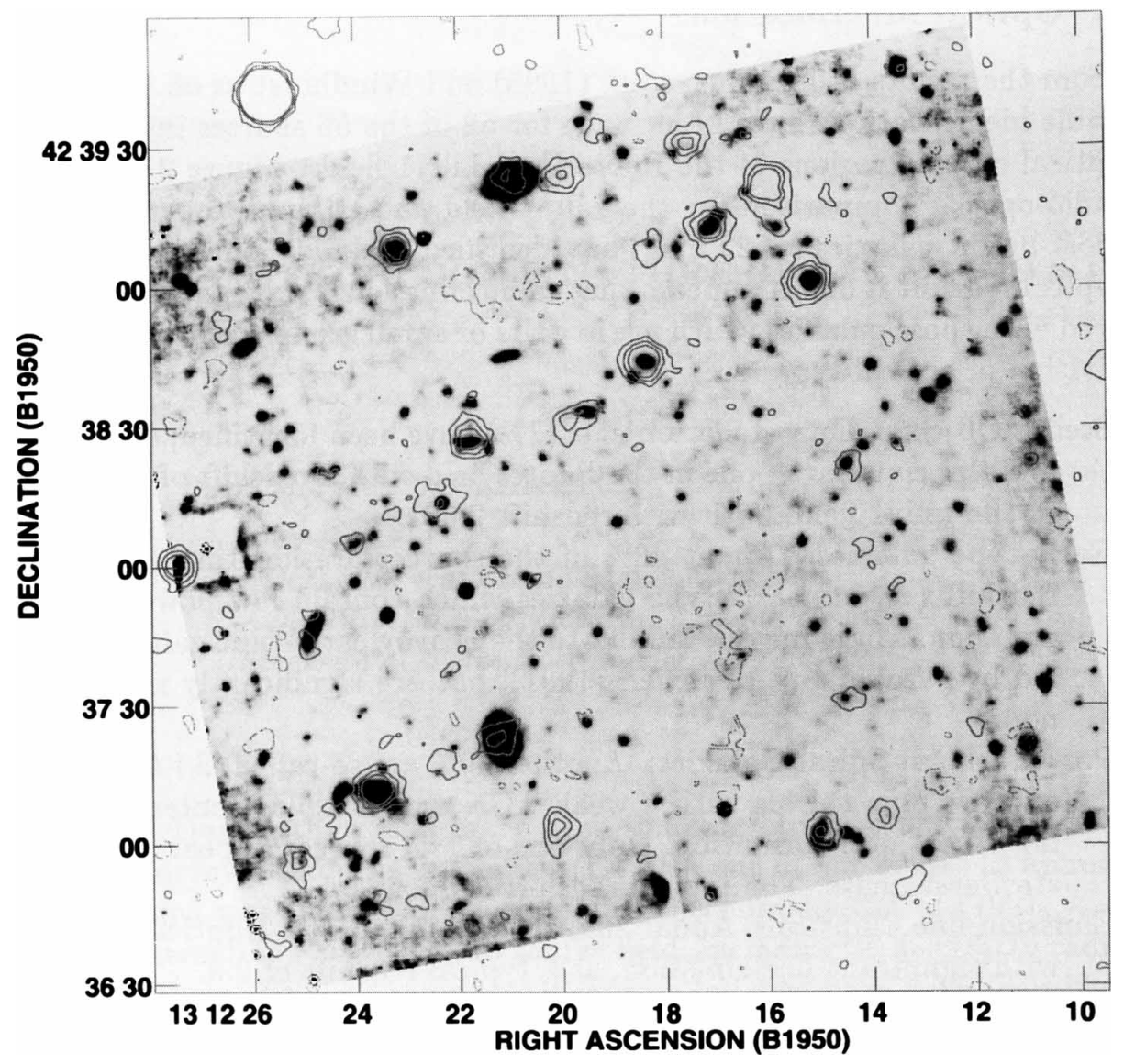

Figure 2. The Lilly1 Field, Radio and Optical: The radio image with 6" resolution is shown by the contour levels at $-3.5,3.5,7.0,10.5,14.0 \mu \mathrm{Jy} /$ beam. The HST WFC I-band image with $0.5^{\prime \prime}$ resolution is shown in grey-scale. Galaxies brighter than $22 \mathrm{~m}$ are saturated, with the fainter galaxies at 26 mag. On close inspection of the radio-optical correspondence, only two radio sources are unidentified.

faint background sources. Thus, most of the sources are less than about $2^{\prime \prime}$ in angular size. None of the 39 sources appear larger than $10^{\prime \prime}$; perhaps two sources are about $5^{\prime \prime}$ in size.

Limited spectral information is available for the $\mu \mathrm{Jy}$ sources since only the Bootes field was observed at two frequencies. Data in the Lynx and Cepheus field at other frequencies were also available (Windhorst et al. 1993 ). For the $\mu \mathrm{Jy}$ sources the average $\alpha=-0.4$ (where $S \sim \nu^{\alpha}$ ), the same for that of the mJy sources. About $35 \%$ of the sources have steep $(\alpha<$ $-0.5)$ spectra, $20 \%$ have inverted spectra $(\alpha>0.3)$, with the remainder flat spectra. These proportions should be regarded as preliminary. 


\section{Optical Identifications}

From the results of Hammer et al. (1995) and Windhorst et al. (1995), reliable identifications have been made for 52 of the 56 sources in the radiooptical overlap regions of the Bootes and Lilly1 fields. Figure 2 shows the radio-optical comparison for the Lilly1 field. A striking property is that most bright galaxies ( $<22 \mathrm{mag}$ ) are identified with $\mu \mathrm{Jy}$ radio sources, the typical redshift is 0.5 . It appears that radio emission is preferentially associated with those galaxies which are in pairs or small groups. The preliminary division of optical types are:

Stellar Objects: Four stellar objects (7\%) have been identified; one M star and three quasars-one in the Bootes field with a redshift of 0.985 ; two in the Lilly1 field, both with redshift 2.561 .

Early-type galaxies: About $40 \%$ of the sources are identified with high redshift $(z \geq 1)$ early-type galaxies which contain low powered AGN emission. These may be similar to the nearby early-type galaxies studied by Wrobel and Heeschen (1991), but are significantly more luminous.

Post-starburst Spiral Galaxies: Another $30 \%$ are so-called ' $\mathrm{S}+\mathrm{A}$ ' galaxies and are blue spirals with a weak AGN and a stellar content indicating recent star formation. Only one or two sources appear to be in a starburst phase. The typical redshift is about 0.5 .

Emission-line Ellipticals: About $20 \%$ are associated with elliptical galaxies, with significant line emission, at a typical redshift of 0.8 .

Observations with many telescopes at several wavelengths will continue in order to determine the properties of these faint radio sources which are an important constituent in the early universe.

Others contributing to these results are K.I.Kellermann (NRAO), R.B. Partridge (Haverford College), E. Richards (University of Virginia), and R. Windhorst (Arizona State University).

\section{References}

Fomalont, E.B., Windhorst, R.A., Kristian, J.A., \& Kellermann, K.I. 1991, AJ, 102, 1258 Hammer, F., Crampton, D., Lilly, S.J., LeFevre, O., \& Kenet, T. 1995 MNRAS, 276, 1085

Richards, E. 1996, in preparation.

Windhorst, R.A., Fomalont, E.B., Kellermann, K.I., Partridge, R.A., Richards, E., Franklin, B.E., Pascarelle, S.M., \& Griffiths, R.E. 1995, Nature, 471

Windhorst, R.A., Fomalont, E.B., Partridge, R.B., \& Lowenthal, J.D. 1993, ApJ, 498

Wrobel, J.M., Heeschen, D.S. 1991, AJ, 101, 148 